\title{
Calibrating Wavelengths in the Region From 0.6 to 2.6 Microns
}

\author{
Nicolo Acquista and Earle K. Plyler
}

\begin{abstract}
The wavelengths of twenty absorption bands have been measured on a grating spectrometer in the region from 0.6 to 2.6 microns. Also, several emission lines of krypton hav? been measured in the near infrared region. The purpose of this investigation was to make available additional calibration points for prism instruments.

The bands which were selected for calibration are parts of the absorption spectra of didymium glass, carbon disulfide, 1,2,4-trichlorobenzene, carbon disulfide, and polystyrene. Five graphs of the measured spectra are included, and the calibrated waveiengths are marked on the bands in order to facilitate identification.
\end{abstract}

In order to calibrate prism instruments adequately, many absorption bands and emission lines of accurately known wavelengths are needed. This is especially so in the wavelength region between 0.6 to $2.6 \mu$, where the wave number span is $16,000 \mathrm{~cm}^{-1}$. The purpose of this investigation was to provide additional calibration points in the near infrared region for use with prism instruments. Previously, the problem of obtaining calibration points in the infrared region has been studied by Oetjen. ChaoLou Kao, and Randall. ${ }^{1}$ More recently, Plyler and Peters $^{2}$ have added calibration points covering the region from 2 to $25 \mu$. There are still several gaps where further calibration points would be highly desirable, in addition to the combined results given in the articles cited in footnotes 1 and 2 .

The calibration of other points in the near infrared region from the visible to $2.5 \mu$ has been neglected because no fund amental vibrational absorption bands have wavelengths less than $2.5 \mu$. For this reason, most observations commence at $2 \mu$. Recently, the importance of combination and harmonic bands has been recognized in the analysis of spectra, and measurements are now being made at wavelengths less than $2 \mu$. More accurate calibrating wavelengths are necessary in order to fully utilize the high sensitivity of instruments which are equipped with lead sulfide $(\mathrm{PbS})$ cells. The lead sulfide cell has been found to be very sensitive for the detection of radiant energy from 0.6 to $2.6 \mu$ and thus allows a much greater accuracy and higher resolution than hitherto possible for prism instruments. The wavelengths listed in the present study should assist in relieving this situation.

The absorption bands selected for calibration were measured on a high-dispersion grating spectrometer. Thespectrometerhas a 15,000-line-per-inch grating as the dispersing unit, and the collimating mirror has a focal length of $102 \mathrm{~cm}$. A lead-sulfide photoconducting cell detects the radiant energy passing through the exit slit of the spectrometer. A more complete description of the grating spectrometer has been given in a previous paper and will not be repeated here. ${ }^{3}$

R. A Oetjen, Chao-Lou Kao, and H . M Randall, Rev Sci. Instr. 13, 515 (1942)

2 Earle K. Plyler and C. Wilbur Peters, J. Research NBS 45, 462 (1950) R P2153. 3 Earle K. Plyler and Norman Gailar, J. Research NBS 4\%, 248 (1951) RP2249.
The calibration of the spectromeier has been made by the use of higher orders of standard atomic lines that appear as first order lines in the visible and near infrared spectrum. The emission lines from mercury, krypton, argon, neon, and xenon were observed from high current ares, and the position of the grating was indicated by a counter when the lines were detected. This made $i_{i}$ possible to obtain a wavelength scale as a function of the counter readings. By the use of the calibration curve for the grating spectrometer, it was found that the wavelengths of spectral lines can be measured to an accuracy of $\pm 0.0001 \mu$. When absorption bands are measured, the wavelengths can usually be determined to an accuracy of $\pm 0.001 \mu$. The grating spectrometer has a high dispersion and the bands are wide, so that there is an uncertainty in locating the position of maximum absorption. This reduces the accuracy of the wavelengths to the above mentioned value. In order to have a check on the wavelength calibration, two sources of radiation are usually recorded simultaneously. One source is a tungsten-ribbonfilament lamp, which is operated with a current of 35 amperes and serves as the radiator of the continuous energy. The other source is a mercury or a krypton arc. By means of mirrors, both sources are focussed on the entrance slit of the spectrometer.

The experimental results are represenced in figures 1 to 5, and show the spectra of didymium glass, polystyrene, 1,2,4-trichlorobenzene, and the emission spectrum of krypton. The didymium glass was selected on the basis of its successful use for calibrations in the visible region, where tests over a number of years have shown that the bands do not change in position with different batches. The polystyrene and 1,2,4-trichlorobenzene were selected because they are now in use in the longer wavelength region; thus the total number of materials needed for calibration would not be increased. One band of carbon disulfide $\left(\mathrm{CS}_{2}\right)$ with a wavelength of $2.224 \mu$ has been calibrated. It is well separated from the other bands of $\mathrm{CS}_{2}$ and no figure is given as there should be little difficulty in its identification.

Figure 1 is a trace of the spectrum of didymium glass as measured on a model 21 Perkin-Elmer spectrometer containing a $\mathrm{NaCl}$ prism. The wavelengths measured by the use of the grating spectrometer are marked on the figure, and are also listed in table 1. 
TABLE 1. Calibration wavelengths for prism instruments

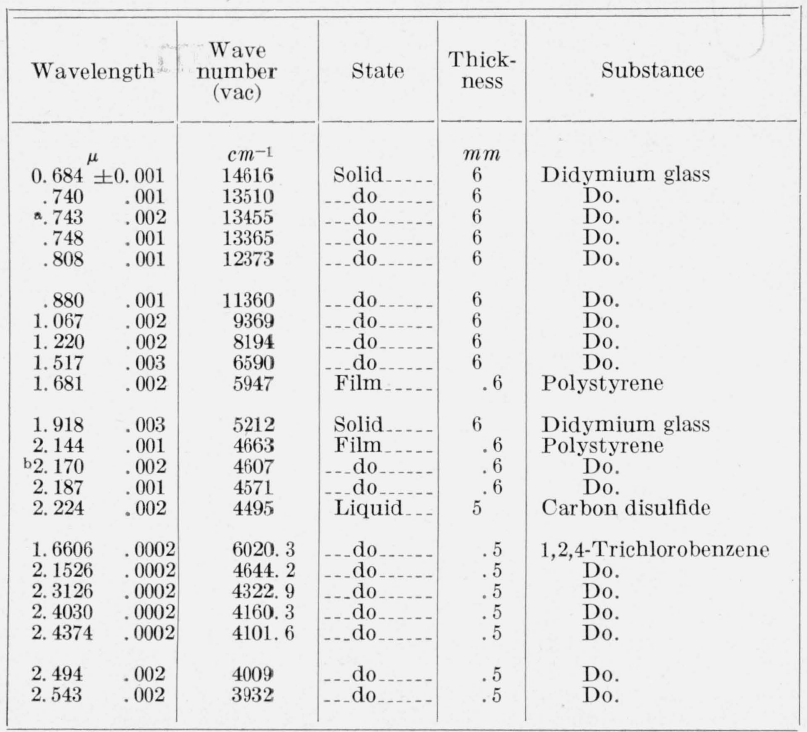

a A verage value of the maxima for 0.740 and $0.748 \mu$.

b Principal band observed with prism spectrometer.

Figure 2 is a trace of the record obtained for the two bands at 0.743 and $0.808 \mu$ under high resolution. The emission lines are produced by a mercury arc. The wavelengths listed on the graph for these lines are given as twice the standard values since they are recorded in the second order. Figure 3 represents part of the spectrum of polystyrene and shows the regions used for calibration. The $1.681-\mu$ band is composed of one component, but the $2.170-\mu$ band has two side branches that are hardly noticeable on the prism instrument. Figure 4 represents the spectrum of 1,2,4-trichlorobenzene from 1.6606 to 2.543 $\mu$. The wavelengths are marked on the bands which have been calibrated. In a previous publication (see footnote 2 ) the bands with wavelengths 2.494 and $2.543 \mu$ were not labeled; two other bands were incorrectly labeled with these wavelengths. A glass prism was used in the spectrometer for the measurement of the emission spectrum of krypton, the results being represented by figure 5. 'The width of some of the observed lines indicated that several components might be present. Further measurements with the grating spectrometer proved that all the lines measured by the prism instrument had several components.

All of the calibrating wavelengths determined in the present study are listed in tables 1 and 2 .

The position of the maximum of absorption of those bands, which are not symmetrical or which have two or more components, is usually changed when meas-

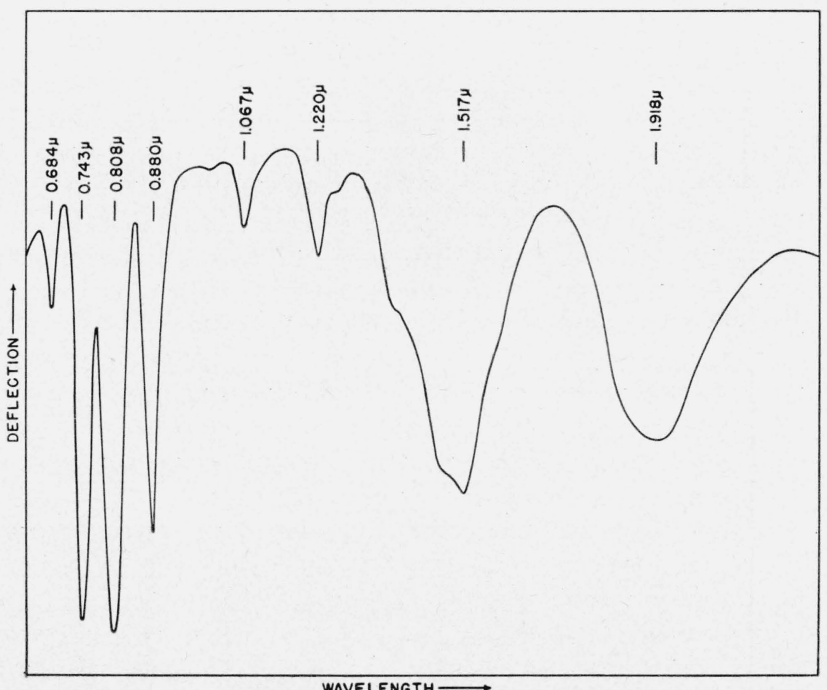

FIGURE 1. The adsorption spectrum of didymium glass under prism dispersion from 0.6 to $2.0 \mu ; t=0.6 \mathrm{~mm}$.

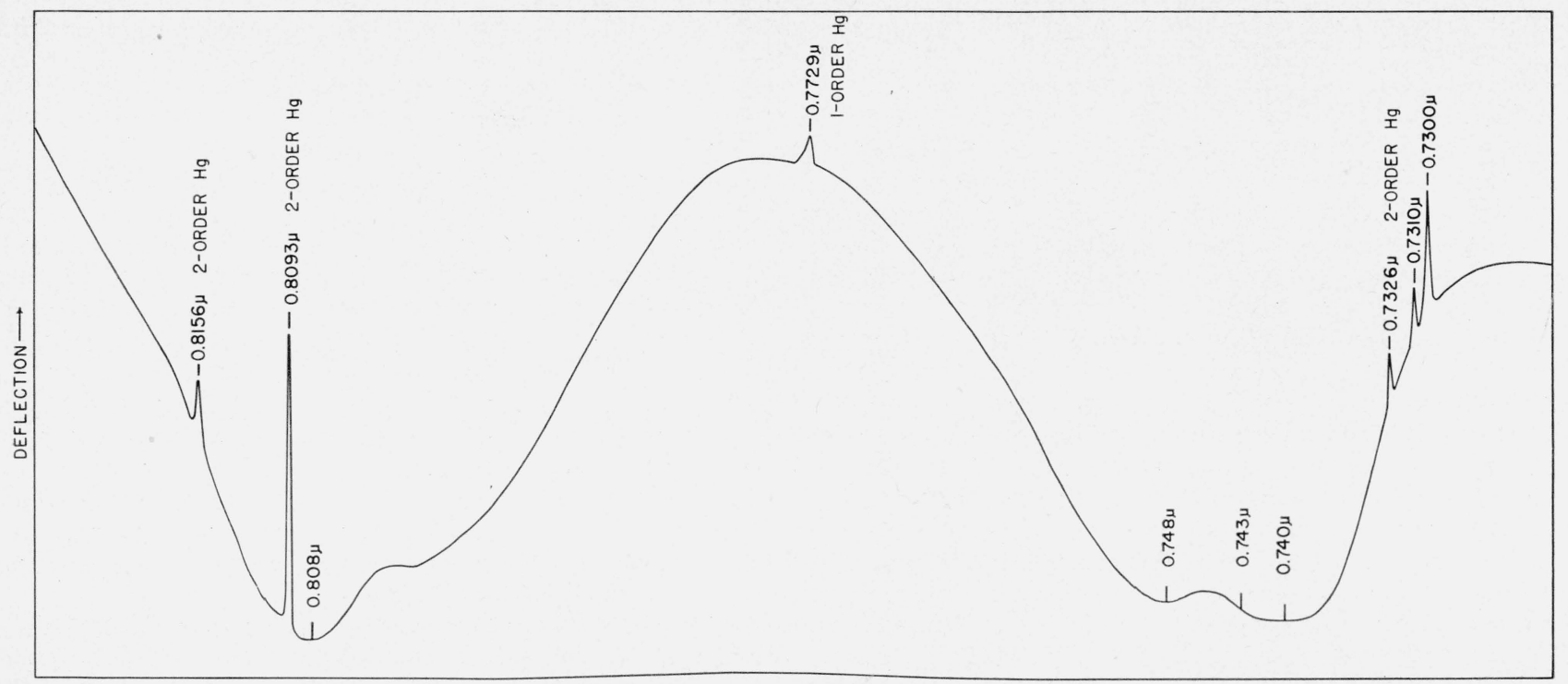

FIGURE 2. The absorption spectrum of didymium glass with mercury lines superimposed as obtained with a grating instrument for the bands at 0.743 and $0.808 \mu ; t=6 \mathrm{~mm}$. 


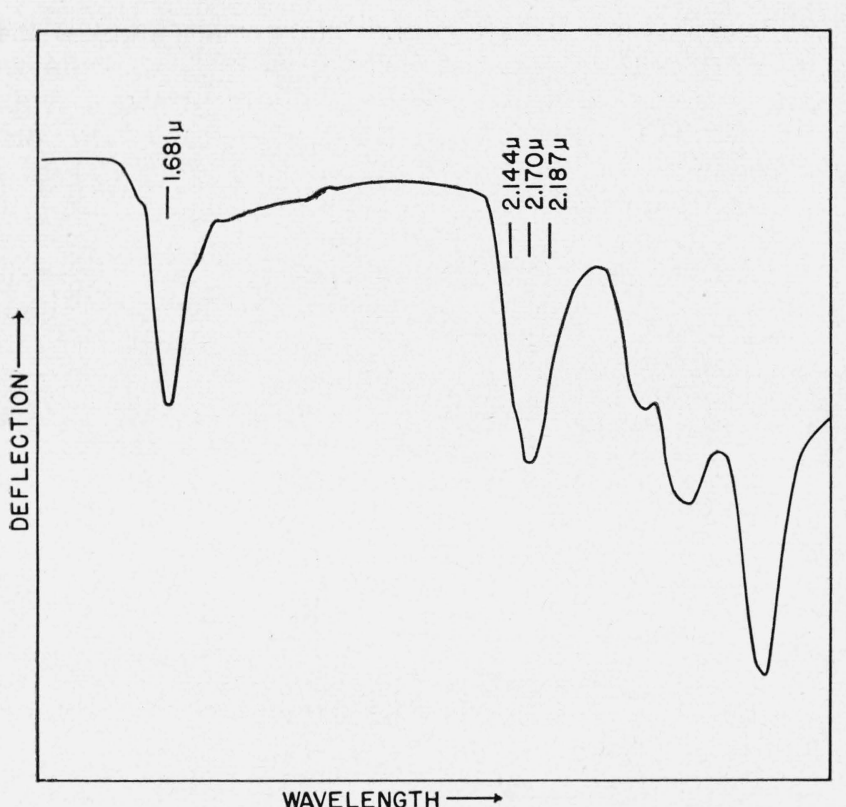

FIGURE 3. The infrared absorption bands of polystyrene at 1.681 and $2.1 \% 0 \mu$ obtained with a prism instrument; $t=0.6$ $\mathrm{mm}$.

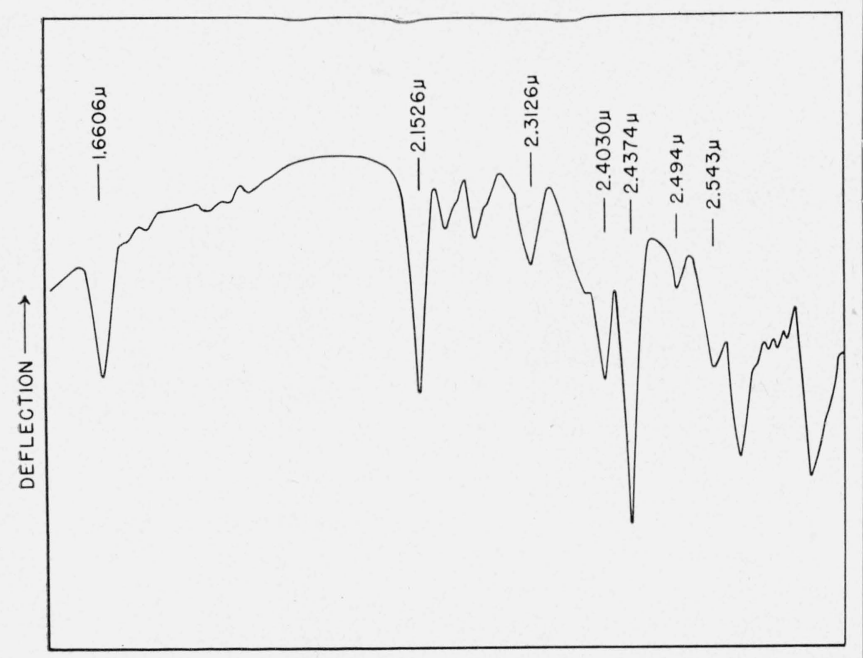

WAVELENGTH

Figure 4. Near infrared spectrum of 1,2,4-trichlorobenzene observed with a lithium fluoride prism; $0.5 \mathrm{~mm}$ cell.

ured with different spectral slit widths. When narrow slits corresponding to a spectral interval of 1 to $3 \mathrm{~A}$ are used, as with the grating spectrometer, the true band shapes are observed and each component is resolved. When spectral slit widths of $100 \mathrm{~A}$ or greater are used, as with prism instruments, a change in wavelength of the maximum of absorption of some bands may occur. This effect has been observed by K. S. Gibson, ${ }^{4}$ who found that the wavelength of maximum absorption of the $0.743-\mu$ band of didymium glass occurred at $0.745 \mu$ when the spectral slit was changed from 100 to $200 \mathrm{~A}$.

The calibration wavelengths of the bands of didymium glass in the region of 0.68 to $0.88 \mu$, which are

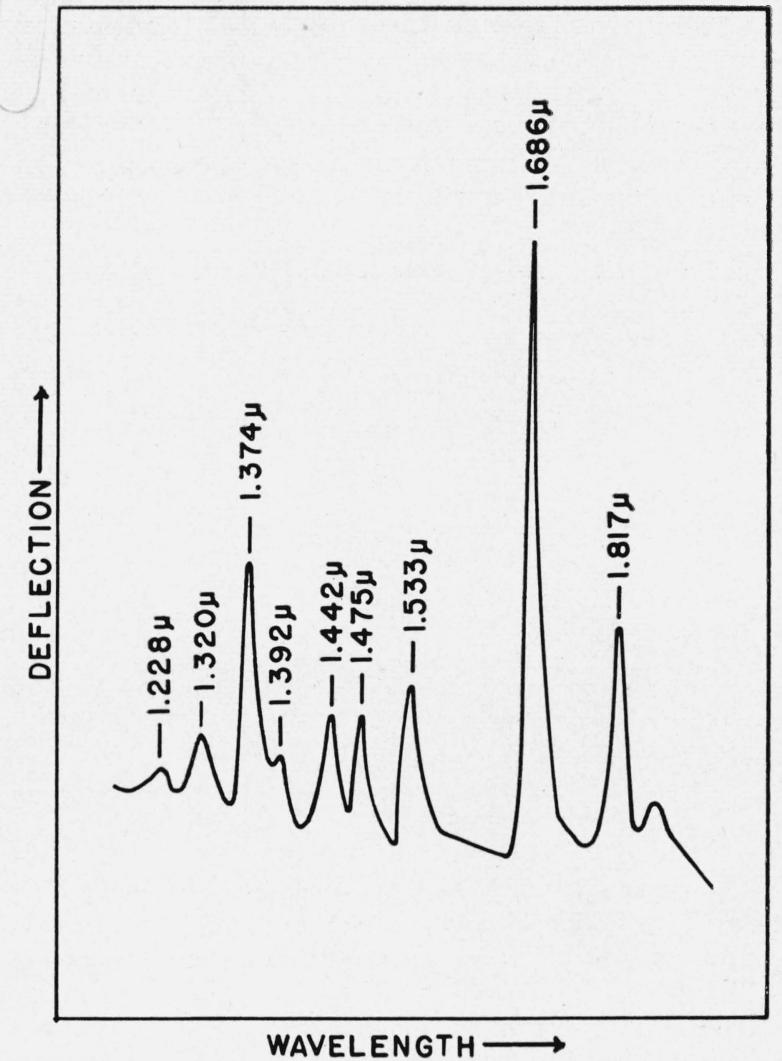

FIGURE 5. Infrared emission lines of krypton obtained with a Perkin-Elmer spectrometer with a glass prism.

listed in table 1 , have been compared with the measurements of Gibson. ${ }^{4}$ Of the six bands, which were measured independently, the wavelength value of only one differs by more than $\pm 0.001 \mu$. This is within the probable error of measurement. In this study the wavelength of one band has been found to be $0.880 \mu$, whereas Gibson reports a value of $0.883 \mu$.

TABLE 2. Calibrated krypton lines for prism instruments

\begin{tabular}{|c|c|c|c|c|c|}
\hline $\begin{array}{l}\text { Wavelength } \\
\text { prism value }\end{array}$ & $\begin{array}{c}\text { Wave } \\
\text { number } \\
\text { (vac) }\end{array}$ & $\begin{array}{l}\text { Wave- } \\
\text { length grat- } \\
\text { ing value }\end{array}$ & $\begin{array}{l}\text { W avelength } \\
\text { prism value }\end{array}$ & $\begin{array}{c}\text { Wave } \\
\text { number } \\
\text { (vac) }\end{array}$ & $\begin{array}{l}\text { Wave- } \\
\text { length } \\
\text { grating } \\
\text { value }\end{array}$ \\
\hline$\mu$ & $\mathrm{cm}^{-1}$ & $\stackrel{\mu}{\mu}$ & $\mu$ & $\mathrm{cm}^{-1}$ & $\stackrel{\mu}{\mu}$ \\
\hline $\begin{array}{l}1.278 \pm 0.002 \\
1.321 \pm 0.001\end{array}$ & 7538 & $\begin{array}{l}\left\{\begin{array}{l}1.29345 \\
\left\{\begin{array}{l}1.32103 \\
1.33042\end{array}\right.\end{array}\right.\end{array}$ & 1. $475 \pm 0.005$ & 6777 & $\left\{\begin{array}{l}1.47345 \\
1.47628 \\
1.47650 \\
1.49618\end{array}\right.$ \\
\hline $1.374 \pm 0.001$ & 7270 & $\left\{\begin{array}{l}1.37389 \\
1.37633\end{array}\right.$ & & & $\left(\begin{array}{l}1.52399 \\
1.53269\end{array}\right.$ \\
\hline $1.392 \pm 0.003$ & 7182 & $\left\{\begin{array}{l}1.33826 \\
1.33240 \\
1.39331 \\
1.39742\end{array}\right.$ & 1. $533 \pm 0.005$ & 6521 & $\left\{\begin{array}{l}1.53353 \\
1.53719 \\
1.54333 \\
1.54739\end{array}\right.$ \\
\hline $1.442 \pm 0.003$ & 6933 & $\left\{\begin{array}{l}1.44026 \\
1.44239 \\
1.44693\end{array}\right.$ & $1.683 \pm 0.005$ & 5929 & $\left\{\begin{array}{l}1.07800 \\
1.67847 \\
1.68535 \\
1.68904 \\
1.69356\end{array}\right.$ \\
\hline & & & $1.817 \pm 0.001$ & 5502 & $\left\{\begin{array}{l}1.81671 \\
1.81844\end{array}\right.$ \\
\hline
\end{tabular}

4 K. S. Gibson, NBS Circular 484 (1949). 
In table 2 are given the wav elengths of the krypton emission lines as measured by a prism instrument and by the grating spectrometer. The grating values were furnished by C. J. Humphreys ${ }^{5}$ of this Bureau. These lines are not well suited for the calibration of prism instruments with a thermocouple or bolometer

${ }_{5}^{5}$ C. J. Humphreys, private communication. as the detector, but a $\mathrm{p}_{\mathrm{I}}$ ism instrument with a $\mathrm{PbS}$ detector would have sufficient resolution to separate many of the component lines of the krypton spectrum. These lines would be useful for calibration because of their high accuracy, which is about equal to 1 part in a hundred thousand.

Washington, April 2, 1952. 